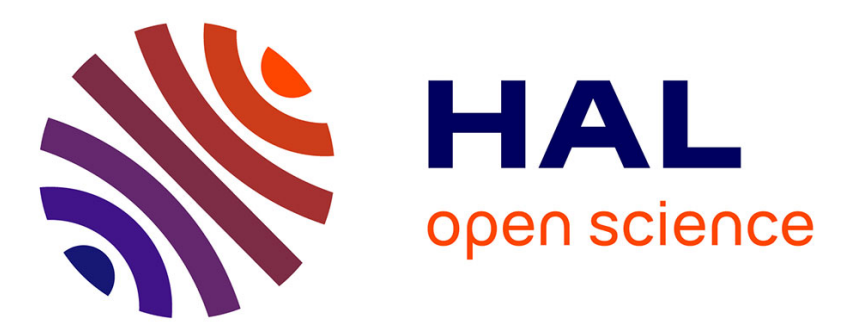

\title{
Transverse vibrations of a thin rectangular porous plate saturated by a fluid
}

\author{
Philippe Leclaire, Kirill Horoshenkov, Alan Cummings
}

\section{To cite this version:}

Philippe Leclaire, Kirill Horoshenkov, Alan Cummings. Transverse vibrations of a thin rectangular porous plate saturated by a fluid. Journal of Sound and Vibration, 2001, 247 (1), pp.1-18. 10.1006/jsvi.2001.3656 . hal-01326042

\section{HAL Id: hal-01326042 \\ https://hal.science/hal-01326042}

Submitted on 2 Jun 2016

HAL is a multi-disciplinary open access archive for the deposit and dissemination of scientific research documents, whether they are published or not. The documents may come from teaching and research institutions in France or abroad, or from public or private research centers.
L'archive ouverte pluridisciplinaire HAL, est destinée au dépôt et à la diffusion de documents scientifiques de niveau recherche, publiés ou non, émanant des établissements d'enseignement et de recherche français ou étrangers, des laboratoires publics ou privés. 


\title{
TRANSVERSE VIBRATIONS OF A THIN RECTANGULAR POROUS PLATE SATURATED BY A FLUID
}

\author{
P. Leclaire and K. V. Horoshenkov \\ Department of Civil and Environmental Engineering, University of Bradford, Bradford BD7 1 DP, England
}

\section{A. Cummings}

School of Engineering, University of Hull, Hull HU6 7 RX, England

\begin{abstract}
A simple model of the transverse vibrations of a thin rectangular porous plate saturated by a fluid is proposed. The model is based on the classical theory of homogeneous plates and on Biot's stress-strain relations in an isotropic porous medium with a uniform porosity. Two coupled dynamic equations of equilibrium relating the plate deflection and the fluid/solid relative displacement are found and their physical interpretation is given. The energy dissipation by viscous friction is included in the model. An approximate calculation of the natural frequencies of vibration is given for rigid plates with various boundary conditions at the edges. The influence of porosity, tortuosity and permeability on the resonances is studied and a condition of maximum damping involving these parameters is given.
\end{abstract}

\section{INTRODUCTION}

The vibration of porous plates is important in the study of fluid-solid interactions with applications in geophysics or in civil engineering, where porous panels are used as sound absorbers. When the material is saturated by air, the approximation of a rigid frame can generally be made. In this approximation, the solid frame is motionless because it is much heavier and more rigid than air. In reality, however, the solid can vibrate in the vicinity of resonance frequencies, resulting in a strong modification of the surface impedance $[1,2]$. It has been shown [3] that Biot's theory $[4,5]$ can be used to determine the displacement of the solid and its effect on the calculation of the surface impedance of rigid porous layers.

Another approach consists in solving the two-dimensional boundary value problem of the vibration of a porous plate. A rigorous and detailed analytical description of this problem including Biot's equations of poroelasticity has been given by Theodorakopoulos and Beskos [6]. In the present article, an additional assumption valid for thin plates is made and an alternative form of the stress-strain relations introduced by Biot $[7,8]$ is used. In this formulation, the equations of equilibrium are derived in a simple way without 
a significant loss of generality. Two coupled equations describing the dynamic equilibrium of the plate are then obtained by introducing inertia forces. Energy dissipation by viscous friction is included through the use of a dynamic fluid density. The calculated deflection compares very well with the numerical results of reference [6]. The simplicity of the equations obtained gives a better understanding of the physical phenomena involved in the vibration. These equations make an evaluation of the natural resonance frequencies with a range of boundary conditions at the edges possible.

In this article, the main results of the classical theory of plates are applied to the non-porous case. This can be justified by the fact that at the scale of the wavelengths the porous medium appears as a continuous medium composed of an effective solid and an effective fluid in Biot's approach of poroelasticity. The plate is treated as a classical homogeneous plate where elastic, inertial and viscous interactions between the solid and the fluid take place within the pores. These effects are described by Biot's coupled equations of motion. The continuity conditions on the pressures and velocities at the interface between the porous surfaces and the surrounding fluid were given by Deresiwicz and Skalak [9]. These are fairly simple and involve the porosity factor. From these conditions, it is possible to model the effect of fluid loading on the vibration. The fluid behaviour in the pores is at the centre of this article and so it is natural and coherent to include the effect of fluid loading at the interfaces. It is thought that the fluid loading will have less effect for porous plates than for non-porous plates, though, because porous media are generally permeable. The effect of fluid loading is studied in the baffled case by the application of the continuity conditions. For simplicity, this effect is not implemented numerically. Attention is particularly focussed on the influence of permeability, porosity and tortuosity.

\section{STRESS-STRAIN RELATIONS FOR A POROUS PLATE}

\subsection{BIOT'S STRESS-STRAIN RELATIONS IN AN INFINITE POROUS MEDIUM}

The stress-strain relations for an unbounded porous medium have been established by Biot. For an isotropic medium with uniform porosity and in the absence of body force, they can be written in the form [8]

$$
\begin{gathered}
\sigma_{i j}+\alpha P_{f} \delta_{i j}=2 \mu \varepsilon_{i j}+\lambda \varepsilon \delta_{i j}, \\
P_{f}=-\alpha M \varepsilon+M \zeta
\end{gathered}
$$

where

$$
\begin{gathered}
\varepsilon_{i j}=\frac{1}{2}\left(\frac{\mathrm{d} u_{i}}{\mathrm{~d} x_{j}}+\frac{\mathrm{d} u_{j}}{\mathrm{~d} x_{i}}\right), \\
\varepsilon=\varepsilon_{11}+\varepsilon_{22}+\varepsilon_{33}=\operatorname{div} \mathbf{u}, \\
\zeta=-\operatorname{div} \mathbf{w}, \quad \mathbf{w}=\phi(\mathbf{U}-\mathbf{u}) .
\end{gathered}
$$

In these equations, $\sigma_{i j}$ is the total stress tensor acting in the porous medium, $\delta_{i j}$ is the Kronecker symbol (the convention of summation on repeated indices being implied), $P_{f}$ is the fluid pressure in the pores, $\lambda$ and $\mu$ are the Lamé elastic constants of the solid frame defined for isotropic media. The coupling coefficient $\alpha$ and the elastic modulus $M$ have been 
defined by Biot [7]. $\varepsilon_{i j}$ is the solid strain tensor expressed as a function of the components of the solid displacement $\mathbf{u}$ in the system of co-ordinates $\left(x_{1}, x_{2}, x_{3}\right), \varepsilon$ is the solid dilatation and the fluid content $\zeta$ is a function of the fluid displacement $\mathbf{U}$ and of the porosity $\phi$, w being the relative fluid-solid displacement.

\subsection{THE BENDING OF A POROUS PLATE}

The condition of equilibrium of a plate under the action of a load or of a buckling constraint can be written in terms of bending and twisting moments related to the deflection (see reference [10]). The problem of a porous plate is treated as follows: in a first step, moments $\mathscr{M}_{1}^{\prime}, \mathscr{M}_{2}^{\prime}$ and $\mathscr{M}_{12}^{\prime}$ are calculated, that are directly related to the deflection of the solid frame but where the first two of these are not the actual bending moments in the fluid-saturated material. Then from a relation obtained for the fluid, it is shown that simple relations can be found between $\mathscr{M}_{1}^{\prime}, \mathscr{M}_{2}^{\prime}, \mathscr{M}_{12}^{\prime}$ and the actual moments. The deflection $w_{s}=u_{3}$ of the plate in the deformed state is considered first (see reference [11] for the relations between deflection, curvatures and bending moments). Since the deflection is undergone by the solid frame, the elements of the strain tensor in the solid can be calculated from the expression for $w_{s}$. It is then easily shown that the corresponding stresses are $\sigma_{i j}+\alpha P_{f}$, the resultant of these stresses in the plate thickness corresponding to $\mathscr{M}_{1}^{\prime}, \mathscr{M}_{2}^{\prime}$ and $\mathscr{M}_{12}^{\prime}$. The advantage of using $\sigma_{i j}+\alpha P_{f}$ is that most of the results obtained in the theory of thin non-porous homogeneous plates can be applied to the porous case.

The dimensions of the plate are $a$ and $b$, and its thickness is $h$. The system of co-ordinates (see Figure 1) is chosen so that the plane defined by the axes $x_{1}$ and $x_{2}$ coincides with the middle surface of the plate before deformation, $x_{1}$ and $x_{2}$ being parallel to the sides $a$ and $b$ respectively. The axis $x_{3}$ is normal to the middle surface. The fluid flow is assumed to be normal to the plane $\left(x_{1}, x_{2}\right)$ so that $\zeta=-\partial \mathbf{w} / \partial x_{3}$ (see reference [12]). The component of $\mathbf{w}$ along $x_{3}$ is written as $w=w_{3}$. The thickness of the plate is taken to be smaller than any acoustic wavelength and the additional assumption is made that the variation of amplitude of the fluid displacement within the plate along $x_{3}$ is small. This allows one to make the same assumption as in the non-porous case on the normal component of the total stress tensor: $\sigma_{33}=0$, valid for fairly rigid thin plates. The deformations in the thickness of the plate are neglected and only the stresses tangent to the surface are considered.

The deflection is undergone by the fluid-saturated plate and by the solid frame in particular. The useful elements of the strain tensor in the solid are therefore (see reference [11])

$$
\varepsilon_{11}=-x_{3} \frac{\partial^{2} w_{s}}{\partial x_{1}^{2}}, \quad \varepsilon_{22}=-x_{3} \frac{\partial^{2} w_{s}}{\partial x_{2}^{2}}, \quad \varepsilon_{12}=-x_{3} \frac{\partial^{2} w_{s}}{\partial x_{1} \partial x_{2}}
$$

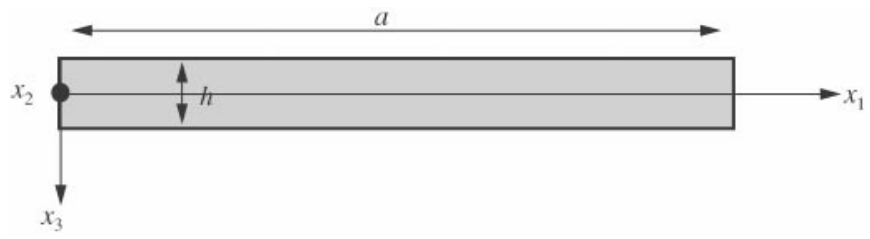

Figure 1. System of co-ordinates in the plate. 
From the formulae of plane elasticity, the corresponding stresses are

$$
\begin{aligned}
\sigma_{11}^{\prime} & =-\frac{E x_{3}}{1-v^{2}}\left(\frac{\partial^{2} w_{s}}{\partial x_{1}^{2}}+v \frac{\partial^{2} w_{s}}{\partial x_{2}^{2}}\right), \\
\sigma_{22}^{\prime} & =-\frac{E x_{3}}{1-v^{2}}\left(\frac{\partial^{2} w_{s}}{\partial x_{2}^{2}}+v \frac{\partial^{2} w_{s}}{\partial x_{1}^{2}}\right), \\
\sigma_{12}^{\prime} & =-\frac{E x_{3}}{1+v}\left(\frac{\partial^{2} w_{s}}{\partial x_{1} \partial x_{2}}\right),
\end{aligned}
$$

where $E$ is Young's modulus and $v$ the Poisson ratio of the solid frame. These expressions are a particular form of Hooke's law. The general form is

$$
\sigma_{i j}^{\prime}=2 \mu^{\prime} \varepsilon_{i j}+\lambda^{\prime} \varepsilon \delta_{i j}
$$

By identifying each term in this equation with those in equation (1) and by the use of the known relations between the elastic constants, it is easily seen that

$$
\begin{gathered}
\sigma_{i j}^{\prime}=\sigma_{i j}+\alpha P_{f} \delta_{i j}, \\
\lambda^{\prime}=\lambda=\frac{E v}{(1+v)(1-2 v)}, \\
\mu^{\prime}=\mu=\frac{E}{2(1+v)} .
\end{gathered}
$$

The stresses $\sigma_{i j}^{\prime}=\sigma_{i j}+\alpha P_{f} \delta_{i j}$ are such that the porous medium behaves as a homogeneous solid of elastic moduli $\lambda$ and $\mu$.

\subsection{RELATIONS BETWEEN THE BENDING MOMENTS}

Two bending moments and one twisting moment may be associated with the stresses $\sigma_{i j}^{\prime}$ given by equation (8),

$$
\begin{aligned}
& \mathscr{M}_{1}^{\prime}=\int_{-h / 2}^{h / 2} \sigma_{11}^{\prime} x_{3} \mathrm{~d} x_{3}=-D\left(\frac{\partial^{2} w_{s}}{\partial x_{1}^{2}}+v \frac{\partial^{2} w_{s}}{\partial x_{2}^{2}}\right), \\
& \mathscr{M}_{2}^{\prime}=\int_{-h / 2}^{h / 2} \sigma_{22}^{\prime} x_{3} \mathrm{~d} x_{3}=-D\left(\frac{\partial^{2} w_{s}}{\partial x_{2}^{2}}+v \frac{\partial^{2} w_{s}}{\partial x_{1}^{2}}\right), \\
& \mathscr{M}_{12}^{\prime}=\int_{-h / 2}^{h / 2} \sigma_{12}^{\prime} x_{3} \mathrm{~d} x_{3}=-D(1-v) \frac{\partial^{2} w_{s}}{\partial x_{1} \partial x_{2}},
\end{aligned}
$$

where $D=E h^{3} / 12\left(12-v^{2}\right)$ is the plate flexural rigidity. The actual moments in the fluid-saturated solid are those associated with the total stresses:

$$
\mathscr{M}_{1}=\int_{-h / 2}^{h / 2} \sigma_{11} x_{3} \mathrm{~d} x_{3}, \quad \mathscr{M}_{2}=\int_{-h / 2}^{h / 2} \sigma_{22} x_{3} \mathrm{~d} x_{3}, \quad \mathscr{M}_{12}=\int_{-h / 2}^{h / 2} \sigma_{12} x_{3} \mathrm{~d} x_{3} .
$$


A moment, relative to the fluid, may equally be defined as [6]

$$
\mathscr{I}=\int_{-h / 2}^{h / 2} P_{f} x_{3} \mathrm{~d} x_{3}
$$

Equation (2) can be written in the form

$$
P_{f}=-\frac{\alpha M}{E}(1-v)\left(\sigma_{11}^{\prime}+\sigma_{22}^{\prime}\right)+M \zeta
$$

Multiplying each term of this equation by $x_{3}$ and integrating over the thickness yields

$$
\mathscr{I}=-\beta\left(\mathscr{M}_{1}^{\prime}+\mathscr{M}_{2}^{\prime}\right)-M \int_{-h / 2}^{h / 2} \frac{\partial w}{\partial x_{3}} x_{3} \mathrm{~d} x_{3}
$$

with $\beta=\alpha M(1-v) / E$. The second term on the right-hand side could be integrated by parts but is neglected under the assumption on the amplitude of the fluid displacement. It can be seen from equation (10) that

$$
\mathscr{M}_{1}^{\prime}=\mathscr{M}_{1}+\alpha \mathscr{I}, \quad \mathscr{M}_{2}^{\prime}=\mathscr{M}_{2}+\alpha \mathscr{I}, \quad \mathscr{M}_{12}^{\prime}=\mathscr{M}_{12}
$$

and by using equation (17), the following relations are found between the moments:

$$
\begin{gathered}
\mathscr{M}_{1}=(1+\alpha \beta) \mathscr{M}_{1}^{\prime}+\alpha \beta \mathscr{M}_{2}^{\prime}, \\
\mathscr{M}_{2}=\alpha \beta \mathscr{M}_{1}^{\prime}+(1+\alpha \beta) \mathscr{M}_{2}^{\prime}, \quad \mathscr{M}_{12}=\mathscr{M}_{12}^{\prime}, \\
\mathscr{I}=-\beta\left(\mathscr{M}_{1}^{\prime}+\mathscr{M}_{2}^{\prime}\right) .
\end{gathered}
$$

\section{DYNAMIC EQUILIBRIUM}

The case of vibration without energy dissipation is considered first. In this case, only elastic and kinetic energies are involved, the elastic energy being responsible for potential forces within the plate while the kinetic energy is the origin of inertia forces. From the expression given by Biot [8] for an unbounded porous medium, the kinetic energy of the plate is expressed as

$$
\mathscr{T}=\frac{1}{2} \int_{V}\left(\rho \dot{w}_{s}^{2}+2 \rho_{f} \dot{w}_{s} \dot{w}+m \dot{w}^{2}\right) \mathrm{d} V=\frac{h}{2} \int_{S}\left(\rho \dot{w}_{s}^{2}+2 \rho_{f} \dot{w}_{s} \dot{w}+m \dot{w}^{2}\right) \mathrm{d} S,
$$

where $\rho, \rho_{f}$ and $m$ are, respectively, the density of the fluid-solid mixture, the density of the fluid and a mass parameter that can be related to the density of the fluid with the help of the tortuosity $\tau_{\infty}$. The integration is performed either over the volume $V$ of the plate or over the middle surface $S$. The elastic energy is given by

$$
\mathscr{U}=\frac{1}{2} \int_{V}\left(\sigma_{11} \varepsilon_{11}+\sigma_{22} \varepsilon_{22}+\sigma_{12} \varepsilon_{12}+P_{f} \zeta\right) \mathrm{d} V
$$


It can easily be seen that

$$
\mathscr{U}=\frac{1}{2} \int_{V}\left(\sigma_{11}^{\prime} \varepsilon_{11}+\sigma_{22}^{\prime} \varepsilon_{22}+\sigma_{12}^{\prime} \varepsilon_{12}\right) \mathrm{d} V
$$

The equations of dynamic equilibrium can be obtained from Hamilton's principle, that involves minimizing a function of the Lagrangian, the mechanical action, between two instants $t_{0}$ and $t_{1}$ (see reference [11]). The method preferred here consists in equating the projection of the potential and inertia forces derived from the elastic and kinetic energies, along the $x_{1}-, x_{2}$ - and $x_{3}$-axis (see reference [10]). The resultant along $x_{3}$ of all the potential forces acting on and element $\mathrm{d} x_{1} \mathrm{~d} x_{2}$ of the middle surface of the fluid-saturated porous plate is

$$
f_{p}=\frac{\partial Q_{1}}{\partial x_{1}} \mathrm{~d} x_{1} \mathrm{~d} x_{2}+\frac{\partial Q_{2}}{\partial x_{2}} \mathrm{~d} x_{1} \mathrm{~d} x_{2}+q \mathrm{~d} x_{1} \mathrm{~d} x_{2}
$$

where $Q_{1}=\int_{-h / 2}^{h / 2} \sigma_{13} \mathrm{~d} x_{3}$ and $Q_{2}=\int_{-h / 2}^{h / 2} \sigma_{23} \mathrm{~d} x_{3}$ are the total shearing stresses along $x_{3}$, and $q$ is the load applied to the solid as a boundary condition ( $q$ has the dimensions of pressure). The inertia forces can be obtained by integrating the time derivative (denoted by a dot over the derived quantity) of the generalized momenta per unit volume of the fluid-solid mixture over the plate thickness. Under the assumption on the amplitude of the fluid displacement, the inertia forces may be expressed as

$$
f_{i}=h\left(\rho \ddot{w}_{s}+\rho_{f} \ddot{w}\right) \mathrm{d} x_{1} \mathrm{~d} x_{2} \text {. }
$$

Since the fluid/solid relative displacement is normal to the middle surface, the equilibrium of the moments acting on the mixture along $x_{1}$ and $x_{2}$, respectively, may be written as [10]

$$
\begin{aligned}
& \frac{\partial \mathscr{M}_{12}}{\partial x_{1}}-\frac{\partial \mathscr{M}_{2}}{\partial x_{2}}+Q_{2}=0 \\
& \frac{\partial \mathscr{M}_{21}}{\partial x_{2}}+\frac{\partial \mathscr{M}_{1}}{\partial x_{1}}-Q_{1}=0
\end{aligned}
$$

with $\mathscr{M}_{12}=-\mathscr{M}_{21}$. Equating $f_{p}$ and $f_{i}$, and combining equations (24), (26) and (27) to eliminate $Q_{1}$ and $Q_{2}$ yields the equation of equilibrium in terms of moments,

$$
\frac{\partial^{2} \mathscr{M}_{1}}{\partial x_{1}^{2}}+\frac{\partial^{2} \mathscr{M}_{2}}{\partial x_{2}^{2}}-2 \frac{\partial^{2} \mathscr{M}_{12}}{\partial x_{1} \partial x_{2}}=-q+h\left(\rho \ddot{w}_{s}+\rho_{f} \ddot{w}\right)
$$

or, by making use of equations (19),

$(1+\alpha \beta)\left(\frac{\partial^{2} \mathscr{M}_{1}^{\prime}}{\partial x_{1}^{2}}+\frac{\partial^{2} \mathscr{M}_{2}^{\prime}}{\partial x_{2}^{2}}\right)+\alpha \beta\left(\frac{\partial^{2} \mathscr{M}_{2}^{\prime}}{\partial x_{1}^{2}}+\frac{\partial^{2} \mathscr{M}_{1}^{\prime}}{\partial x_{2}^{2}}\right)-2 \frac{\partial^{2} \mathscr{M}_{12}^{\prime}}{\partial x_{1} \partial x_{2}}=-q+h\left(\rho \ddot{w}_{s}+\rho_{f} \ddot{w}\right)$.

The first equation of equilibrium in terms of deflection is obtained by using expressions (13) for $\mathscr{M}_{1}^{\prime}, \mathscr{M}_{2}^{\prime}$ and $\mathscr{M}_{12}^{\prime}$. A simple expression is found,

$$
(1+\alpha \beta+\alpha \beta v) D \nabla^{4} w_{s}+h\left(\rho \ddot{w}_{s}+\rho_{f} \ddot{w}\right)=q
$$


with $\nabla^{4}=\nabla^{2}\left(\nabla^{2}\right)$ and $\nabla^{2}=\partial^{2} / \partial x_{1}^{2}+\partial^{2} / \partial x_{2}^{2}$. The equation of equilibrium is also referred to as the plate equation. A second relation can be obtained from equation (2). The microscopic forces per unit volume $V$ acting on the fluid along $x_{3}$ are given by

$$
\frac{\partial P_{f}}{\partial x_{3}}=\alpha M\left(\frac{\partial^{2} w_{s}}{\partial x_{1}^{2}}+\frac{\partial^{2} w_{s}}{\partial x_{2}^{2}}\right)-M \frac{\partial^{2} w}{\partial x_{3}^{2}},
$$

while the inertia forces per unit volume are $f_{i}^{\prime}=\rho_{f} \ddot{w}_{s}+m \ddot{w}$. Equating the forces $\left(\partial P_{f} / \partial x_{3}\right) \mathrm{d} V$ and $f_{i}^{\prime} \mathrm{d} V$ and integrating over the thickness yields the second equation of dynamic equilibrium. Under the assumption on the fluid displacement amplitude, the term containing the space derivative of $\zeta$ is neglected throughout the plate and

$$
\alpha M h\left(\frac{\partial^{2} w_{s}}{\partial x_{1}^{2}}+\frac{\partial^{2} w_{s}}{\partial x_{2}^{2}}\right)-h\left(\rho_{f} \ddot{w}_{s}+m \ddot{w}\right)=\Delta P,
$$

where the integration constant $\Delta P=P_{f}(-h / 2)-P_{f}(+h / 2)$ is the pressure difference corresponding to the boundary conditions applied on the fluid at $x_{3}= \pm h / 2$. Upon inserting the expression for $\beta$, the coupled equations of dynamic equilibrium are finally written as

$$
\begin{gathered}
\left(D+\frac{\alpha^{2} M h^{3}}{12}\right) \nabla^{4} w_{s}+h\left(\rho \ddot{w}_{s}+\rho_{f} \ddot{w}\right)=q, \\
\alpha M h \nabla^{2} w_{s}-h\left(\rho_{f} \ddot{w}_{s}+m \ddot{w}\right)=\Delta P .
\end{gathered}
$$

The first equation may be interpreted as the instantaneous elastic response of the fluid-saturated plate while the second describes the relative motion between the solid and the fluid. The elastic interactions are quantified by the terms where the coupling coefficient $\alpha$ appears. The inertial interactions are given by the terms containing the accelerations.

In a real situation, the relative fluid-solid motion within the pores is responsible for an energy dissipation with a phase shift, corresponding to a delayed after-effect [12]. The effects of dissipation by viscous friction should naturally be included in the second equation. They can easily be incorporated by considering the coefficient $m$ to be frequency dependent,

$$
m(\omega)=\frac{\rho_{22}(\omega)}{\phi^{2}}=\frac{\tau(\omega)}{\phi} \rho_{f}
$$

$\omega$ being the angular frequency. The frequency-independent coefficients $m$ and $\rho_{22}$ have been defined by Biot [8]. The harmonic dependence in time is assumed for the displacements, for $q$ and for $\Delta P$. The dynamic tortuosity $\tau(\omega)$ has been introduced by Johnson et al. [13] and can be written as a complex quantity ( $\mathrm{j}$ being the imaginary unit complex number)

$$
\tau(\omega)=\tau_{\infty}-\mathrm{j} \frac{b_{f}}{\phi \rho_{f} \omega} F(\omega),
$$

where $b_{f}$ is Biot's coefficient of friction [4] given as a function of the porosity, of the fluid dynamic viscosity $\eta$ and of the permeability $\kappa$ by

$$
b_{f}=\eta \phi^{2} / \kappa
$$


The viscosity correction function $F(\omega)$ is close to 1 at low frequencies and describes the effects of viscous friction at high frequencies. Various models can be used to evaluate this function $[5,13-16]$.

The solutions of the plate equations are expressed in the form of double infinite series,

$$
\begin{gathered}
w_{s}\left(x_{1}, x_{2}\right)=\sum_{m=1}^{\infty} \sum_{n=1}^{\infty} W_{m n}^{s} \theta_{m}\left(x_{1}\right) \psi_{n}\left(x_{2}\right), \\
w\left(x_{1}, x_{2}\right)=\sum_{m=1}^{\infty} \sum_{n=1}^{\infty} W_{m n} \theta_{m}\left(x_{1}\right) \psi_{n}\left(x_{2}\right),
\end{gathered}
$$

each term of $w_{s}$ and $w$ satisfying equations (33) and (34). $W_{m n}^{s}$ and $W_{m n}$ are the displacement amplitudes at the position $\left(x_{1}, x_{2}\right) . \theta_{m}\left(x_{1}\right)$ and $\psi_{n}\left(x_{2}\right)$ are the so-called beam functions chosen to comply with the boundary conditions at the edges. If the plate is simply supported, the products of the beam functions $\theta_{m}\left(x_{1}\right)=\sin (m \pi / a)$ and $\psi_{n}\left(x_{2}\right)=\sin (n \pi / b)$ satisfy exactly the equations of equilibrium. The excitation terms can also be expressed in the form of double infinite series. Inserting equations (38) and (39) into equations (33) and (34) leads to a system of two equations and two unknown for each value of the couple $(m, n)$. Solving this system provides the coefficients $W_{m n}^{s}$ and $W_{m n}$. The solutions for $w_{S}$ and $w$ are obtained after insertion into equations (38) and (39).

\subsection{FLUID LOADING}

The fluid can have an effect on the vibration of a plate. This effect is accounted for through an additional excitation term or load that must be included in the equations. To evaluate the fluid loading, it is necessary to calculate the surface acoustic pressure:

$$
P\left(x_{1}, x_{2}, 0\right)=-\rho_{f} \omega^{2} \iint_{S} w_{T}\left(x_{1}^{\prime}, x_{2}^{\prime}\right) G\left(x_{1}, x_{2}, 0 ; x_{1}^{\prime}, x_{2}^{\prime}, 0\right) \mathrm{d} S^{\prime}
$$

This integral is often referred to as the Rayleigh integral where $G$ is the Green function associated with the contribution of a surface element $\mathrm{d} S^{\prime}$,

$$
G\left(x_{1}, x_{2}, 0 ; x_{1}^{\prime}, x_{2}^{\prime}, 0\right)=\frac{\mathrm{e}^{-\mathrm{j} k R}}{2 \pi R}
$$

$k=\omega / c_{0}$ is the wavenumber and $R$ is given by

$$
R=\sqrt{\left(x_{1}-x_{1}^{\prime}\right)^{2}+\left(x_{2}-x_{2}^{\prime}\right)^{2}} .
$$

In the present study, the term $w_{T}\left(x_{1}, x_{2}\right)$ is the total velocity field in the vicinity of the surface. The total velocity field is obtained from a study by Deresiewicz and Skalak [9] on the continuity conditions at the interface between two media involving porous materials. The following continuity equation is given on the velocities at the interface separating a porous medium (1) and a fluid (2),

$$
(1-\phi) \dot{u}_{n}+\phi \dot{U}_{n}=\dot{U}_{n},
$$


where $\dot{u}_{n}$ and $\dot{U}_{n}$ are, respectively, the normal components of the solid velocity $\dot{\mathbf{u}}$, of the fluid velocity $\dot{\mathbf{U}}$ in the porous medium (1) and $\overline{\dot{U}}_{n}$ the velocity in fluid (2) averaged over the bulk area.

Upon using this condition and the definition of the fluid-solid relative displacement [8]

$$
\mathbf{w}=\phi(\mathbf{U}-\mathbf{u})
$$

the total velocity field in the vicinity of the surface of the porous plate is given by the simple sum

$$
\dot{w}_{T}\left(x_{1}, x_{2}\right)=\dot{w}_{s}\left(x_{1}, x_{2}\right)+\dot{w}\left(x_{1}, x_{2}\right),
$$

each component of this field being parallel to the $x_{3}$-axis.

The additional terms due to fluid loading can be written as [17]

$$
f_{m n}^{P}=-\iint_{S} P\left(x_{1}, x_{2}, 0\right) \theta_{m}\left(x_{1}\right) \psi_{n}\left(x_{2}\right) \mathrm{d} S,
$$

which can be rewritten in terms of the radiation impedance matrix $Z_{m n p q}^{B F}$ as

$$
f_{m n}^{P}=-\mathrm{j} \omega \sum_{p q} Z_{m n p q}^{B F}\left(w_{T}\right)_{p q}
$$

Inserting equations (38) and (39) into equations (33) and (34) leads to the modal form of the equations of dynamic equilibrium in which the excitation terms $q$ and $\Delta P$ appear as $q_{m n}$ and $\Delta P_{m n}$ respectively. Equation (33) is interpreted as the response of the fluid-saturated plate while equation (34) describes the relative motion between the solid and the fluid and so the fluid loading is accounted for by adding each term $f_{m n}^{P}$ to $q_{m n}$ in the modal form of equation (33).

\subsection{NUMERICAL RESULTS}

The plate deflection $w_{s}$ computed from the present model is compared to the results of Theodorakopoulos and Beskos [6] with the same numerical data for a water-saturated plate of $4 \times 4 \times 0 \cdot 2 \mathrm{~m}$. The model of Theodorakopoulos and Beskos can be considered as more rigorous than the present model because the use of the moment $\mathscr{I}=-\phi \int_{-h / 2}^{h / 2} P_{f} x_{3} \mathrm{~d} x_{3}$ does not imply any restriction on the fluid. Short wavelengths, i.e. several spatial periods of the fluid displacement, are allowed within the plate whereas the amplitude of the fluid-solid relative displacement is considered as constant throughout the plate thickness in the present model. Figure 2 shows the modulus of the deflection calculated at the centre for a $4 \times 4 \times 0 \cdot 2 \mathrm{~m}$ sandstone plate saturated by water (see Table 1 ). The material properties are those of Fatt $[6,18]$. In the simulation, the plate is uniformly excited by an incident pressure of amplitude $P_{0}=1400 \mathrm{~Pa}$, constant in the frequency domain. The results for an air-filled porous panel ("Y foam") of $0.5 \mathrm{~m} \times 0.5 \mathrm{~m} \times 10.70 \mathrm{~mm}$ are given in Figure 3. This plate was fabricated at the University of Bradford from recycled car dashboards for noise control application. Its properties are displayed in Table 1 . The panel is excited either by a uniform incident pressure of $0 \cdot 1 \mathrm{~Pa}$ amplitude or by a unit point force $(1 \mathrm{~N})$ located at $x_{0}=0.1 \mathrm{~m}, y_{0}=0.15 \mathrm{~m}$. The response is calculated in both cases at $x=0.25 \mathrm{~m}, y=0.25 \mathrm{~m}$. 


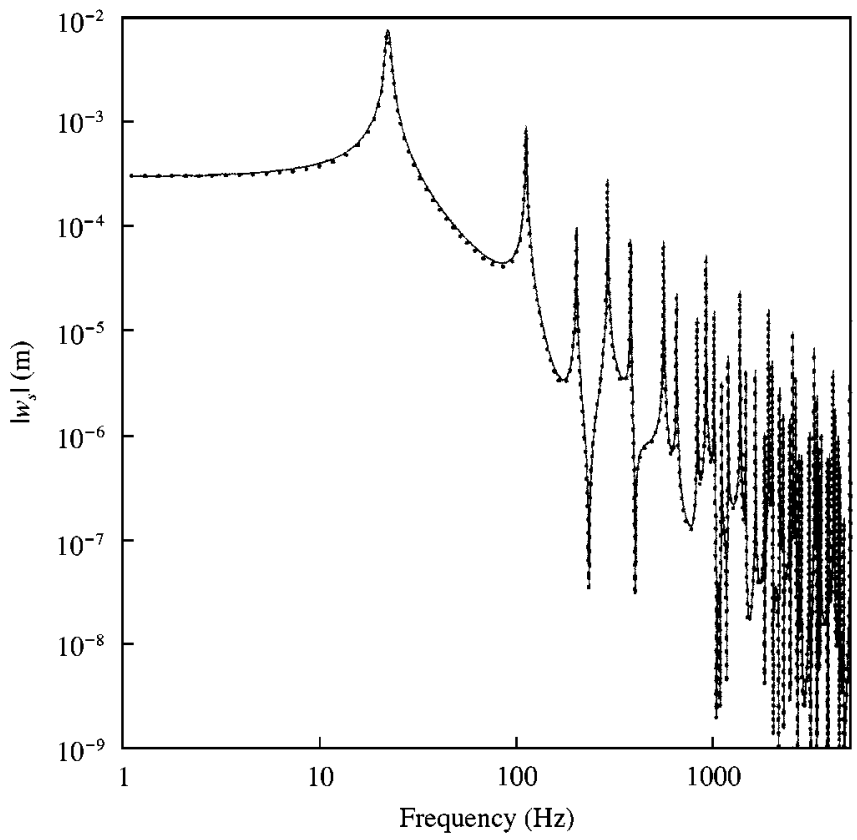

Figure 2. Deflection at the centre of a simply supported water-saturated sandstone excited by a uniform pressure....., Theodorakopoulos and Beskos; —- curve, the present model.

TABLE 1

Plate properties

\begin{tabular}{lcc}
\hline & Sandstone & Y foam \\
\hline Lateral size $a(\mathrm{~m})$ & 4 & $0 \cdot 5$ \\
Lateral size $b(\mathrm{~m})$ & 4 & $0 \cdot 5$ \\
Thickness $h(\mathrm{~mm})$ & 200 & $10 \cdot 70$ \\
Solid density $\rho_{s}\left(\mathrm{~kg} / \mathrm{m}^{3}\right)$ & 2260 & 1136 \\
Young's mod. $E(\mathrm{~Pa})$ & $6 \cdot 9 \times 10^{9}$ & $2 \cdot 1 \times 10^{7}$ \\
The Poisson ratio $v$ & $0 \cdot 25$ & $0 \cdot 35$ \\
Loss factor & 0 & $0 \cdot 1$ \\
Porosity $\phi$ & $0 \cdot 3$ & $0 \cdot 69$ \\
Tortuosity $\tau_{\infty}$ & $1 \cdot 0$ & $1 \cdot 2$ \\
Permeability $\kappa\left(\mathrm{m}^{2}\right)$ & $1 \times 10^{-8}$ & $2 \cdot 7 \times 10^{-10}$ \\
Fluid density $\rho_{f}\left(\mathrm{~kg} / \mathrm{m}^{3}\right)$ & 1000 & $1 \cdot 213$ \\
\hline
\end{tabular}

The curves of Figures 2 and 3 were calculated for values of $(m, n)$ of up to 40 . The results given by the two models are very similar and a difference of only a few percent is observed on the amplitude in a wide frequency range. As shown in Figure 3, the discrepancy between these models seems to increase when the frequency reaches values of around $5 \mathrm{kHz}$. The increasing difference is certainly a consequence of the breakdown of the assumption that the plate is much thinner than any acoustic wavelength. Nevertheless, because porous plates will involve smaller Young's moduli and higher loss factors, many applications and experimental observations will be carried out at low frequencies and this model can be considered as useful. It involves simple equations and allows theoretical developments for more complicated configurations. 


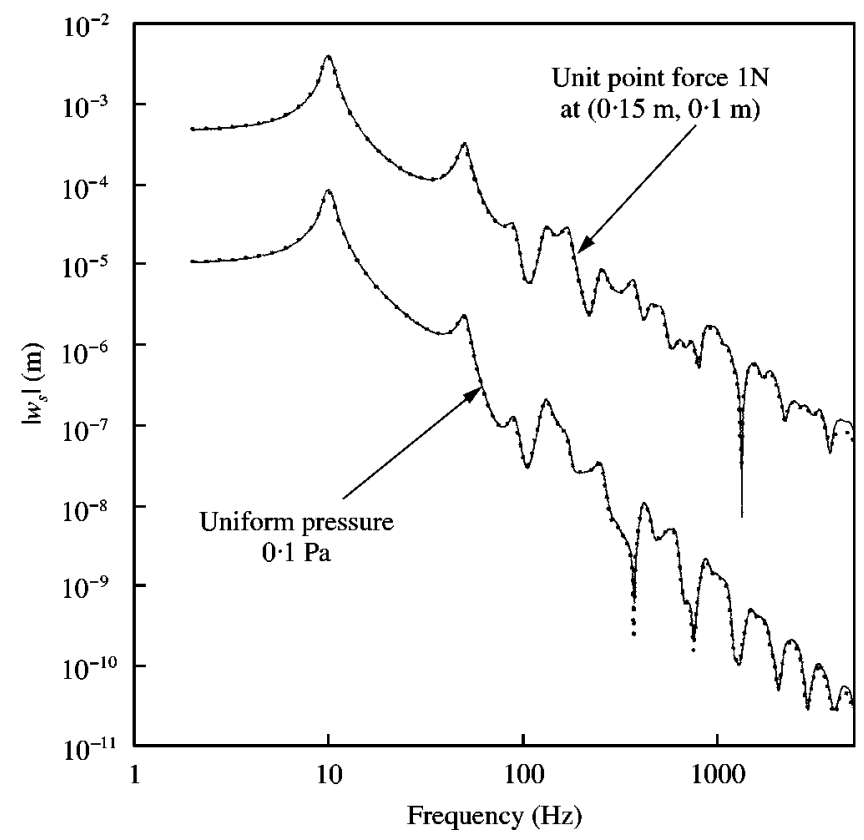

Figure 3. Deflection at the centre of a simply supported air-filled porous panel....., Theodorakopoulos and Beskos; —_, the present model.

\section{APPROXIMATE CALCULATION OF THE NATURAL FREQUENCIES OF VIBRATION OF A POROUS PLATE}

Warburton [19] has provided useful approximate calculations of the natural frequencies of rectangular plates for any combination of the three basic boundary conditions at the edges: i.e., free, simply supported or clamped. Experiments have been carried out in the present investigation to check the accuracy of the prediction on an aluminium plate of $150 \times 50 \times 0.8 \mathrm{~mm}$, with the two a-sides free and the two b-sides clamped. An excellent agreement of less than $3 \%$ error has been obtained for the seven first resonance frequencies.

A similar evaluation can be made for porous materials if the elastic modulus of the plate is much greater than that of the fluid: i.e., if $E \gg M$. This condition will be fulfilled in many cases and in particular for rigid porous plates vibrating in air. In this case, only the inertial interactions are considered in the plate equations, which may be written for free vibration as

$$
D \nabla^{4} w_{s}+h\left(\rho \ddot{w}_{s}+\rho_{f} \ddot{w}\right)=0, \quad \rho_{f} \ddot{w}_{s}+m(\omega) \ddot{w}=0
$$

and which may be combined into a single equation involving only the deflection

$$
D \nabla^{4} w_{s}+h \rho^{\prime} \ddot{w}_{s}=0
$$

with

$$
\rho^{\prime}=\rho-\frac{\rho_{f}^{2}}{m(\omega)}=\rho-\frac{\phi}{\tau(\omega)} \rho_{f}
$$

The case without attenuation is considered first, for the evaluation of the resonance frequencies. In this case, equation (50) indicates that the fluid and the solid vibrate in phase 
with an amplitude ratio of $\left(1-\phi / \tau_{\infty}\right)$. In the restriction stated in this section, the elastic energy is given by

$$
\begin{gathered}
\mathscr{U}=\frac{1}{2} D \int_{S}\left[\left(\frac{\partial^{2} w_{s}}{\partial x_{1}^{2}}\right)^{2}+\left(\frac{\partial^{2} w_{s}}{\partial x_{2}^{2}}\right)^{2}+2 v \frac{\partial^{2} w_{s}}{\partial x_{1}^{2}} \frac{\partial^{2} w_{s}}{\partial x_{2}^{2}}\right. \\
\left.+2(1-v)\left(\frac{\partial^{2} w_{s}}{\partial x_{1} \partial x_{2}}\right)^{2}\right] \mathrm{d} S,
\end{gathered}
$$

the kinetic energy is given by

$$
\mathscr{T}=\frac{1}{2} h \int_{S} \rho^{\prime} \ddot{w}_{s}^{2} \mathrm{~d} S
$$

and, following the method given in reference [19], a frequency factor can be defined as

$$
\begin{aligned}
\Omega^{2} & =\left(\rho-\frac{\phi}{\tau_{\infty}} \rho_{f}\right) \frac{h a^{4}}{\pi^{4} D} \omega^{2}, \\
& =G_{x}^{4}+G_{y}^{4} \frac{a^{4}}{b^{4}}+2 \frac{a^{2}}{b^{2}}\left[v H_{x} H_{y}+(1-v) J_{x} J_{y}\right],
\end{aligned}
$$

giving a value of the natural frequencies for boundary conditions characterized by the values of the coefficients $G_{x}, G_{y}, H_{x}, H_{y}, J_{x}$ and $J_{y}$. A useful table of the values taken by these coefficients for any given set of boundary conditions has been provided by Warburton [19]. For the water-saturated plate of reference [6], the numerical results given by the approximate calculation are close to those provided by the numerical implementation of the model. The eight first natural frequencies calculated from the approximate formula (54) are compared to the corresponding "exact" frequencies obtained from the full model in Table 2 for Y foam. The agreement is excellent except for the sixth frequency. The reason is that the frequency corresponding to $(m, n)=(5,2)$ or $(2,5)$ is $86 \mathrm{~Hz}$. This value is close to that for $(m, n)=(4,4)$. Due to the high loss factor it is not possible to distinguish the two frequencies and only one intermediate value can be read on the curve. As will be shown, the damping by viscous friction can be important at a particular frequency. An evaluation of

\section{TABLE 2}

Comparison of the natural frequencies calculated from the approximate formula (54) with those obtained from the full model

\begin{tabular}{lcc}
\hline & Frequency (approx.) $(\mathrm{Hz})$ & Frequency (theoretical) $(\mathrm{Hz})$ \\
\hline$(2,2)$ & $10 \cdot 1$ & $10 \cdot 0$ \\
$(2,3)$ or $(3,2)$ & $25 \cdot 3$ & $24 \cdot 9$ \\
$(3,3)$ & $40 \cdot 5$ & $39 \cdot 7$ \\
$(4,2)$ or $(2,4)$ & $50 \cdot 6$ & $50 \cdot 1$ \\
$(3,4)$ or $(4,3)$ & $65 \cdot 7$ & $65 \cdot 3$ \\
$(4,4)$ & $91 \cdot 0$ & $88 \cdot 5$ \\
$(5,3)$ or $(3,5)$ & $101 \cdot 2$ & $100 \cdot 9$ \\
$(5,4)$ or $(4,5)$ & $126 \cdot 5$ & $127 \cdot 5$ \\
\hline
\end{tabular}


the resonance frequencies where the viscous friction in the pores are taken into account can be made by using $\operatorname{Re}\left(\rho^{\prime}\right)$ instead of $\rho-\phi \rho_{f} / \tau_{\infty}$ in the expression for $\Omega$. However, the simplicity of the expression for $\omega$ is lost.

\section{INFLUENCE OF POROSITY, TORTUOSITY AND PERMEABILITY ON THE RESONANCES}

The influence of porosity and tortuosity on the resonance frequencies can be studied for $\mathrm{j} b_{f} / \omega \phi \rho_{f}=0$, this imaginary term mainly affecting the quality factor of the resonance. Since the tortuosity is greater than 1 and the porosity is between 0 and 1 , the frequency factor $\Omega$ increases as the porosity decreases or as the tortuosity increases. As a consequence, the resonance frequency will be higher for higher porosities and for lower tortuosities. This is confirmed by the present numerical results and by those of reference [6]. The influence of permeability is studied by reintroducing the coefficient $b_{f}$. The plate equation is then written in a form similar to that of an oscillating system,

$$
D \nabla^{4} w_{s}+h\left(\rho-\frac{\tau_{\infty} \phi \rho_{f}}{\tau_{\infty}^{2}+b_{f}^{2} / \omega^{2} \phi^{2} \rho_{f}^{2}}-\mathrm{j} \frac{b_{f} / \omega}{\tau_{\infty}^{2}+b_{f}^{2} / \omega^{2} \phi^{2} \rho_{f}^{2}}\right) w_{s}=0,
$$

where the expression in parentheses is the dynamic density $\rho^{\prime}$. By considering the real part of equation (55) and from the expression for $\Omega$, it can be observed that the resonance frequency decreases as the ratio $b_{f} / \omega$ increases: i.e., as the permeability decreases.

Equation (55) also shows that increasing the tortuosity or decreasing the porosity $\left(\phi^{2}\right.$ also appears in $b_{f}$ ) reduces the imaginary part and therefore increases the quality factor. Starting from 0 , an increase of the ratio $b_{f} / \omega$ (i.e. a decrease of the permeability), results in an increase in damping, which reaches a maximum value and then goes back to 0 as $b_{f} / \omega$ tends to infinity. In the low frequency range: i.e., for $F(\omega) \simeq 1$, the dynamic density or apparent mass of the plate can be written as

$$
\rho^{\prime}=\rho-\frac{\phi}{\tau_{\infty}} \rho_{f}[R(x)+\mathrm{j} I(x)]
$$

where the function $R(x)+\mathrm{j} I(x)$ gives the change in apparent fluid density due to the motion. This function, given by

$$
R(x)=\frac{x^{2}}{1+x^{2}}, \quad I(x)=\frac{x}{1+x^{2}}
$$

where $x=\omega \tau_{\infty} \phi \rho_{f} / b_{f}$ is plotted in Figure 4 as a function of the dimensionless frequency $x$. The maximum of $I(x)$ is $\frac{1}{2}$ and is reached for $x=x_{0}=1$. As a consequence, the frequency for which the damping by viscous friction is maximum is given by

$$
\omega_{0}=\frac{b_{f}}{\tau_{\infty} \phi \rho_{f}}=\frac{\eta \phi}{\kappa \tau_{\infty} \rho_{f}} .
$$

The permeability affects only $\omega_{0}$ while the porosity and the tortuosity affect both $\omega_{0}$ and the maximum value of the imaginary part of $\rho^{\prime}$. This frequency is almost identical to Biot's characteristic frequency with the difference that it accounts for the tortuosity. It has been introduced by Dunn [20], with a qualitative justification, in the study of the low frequency 


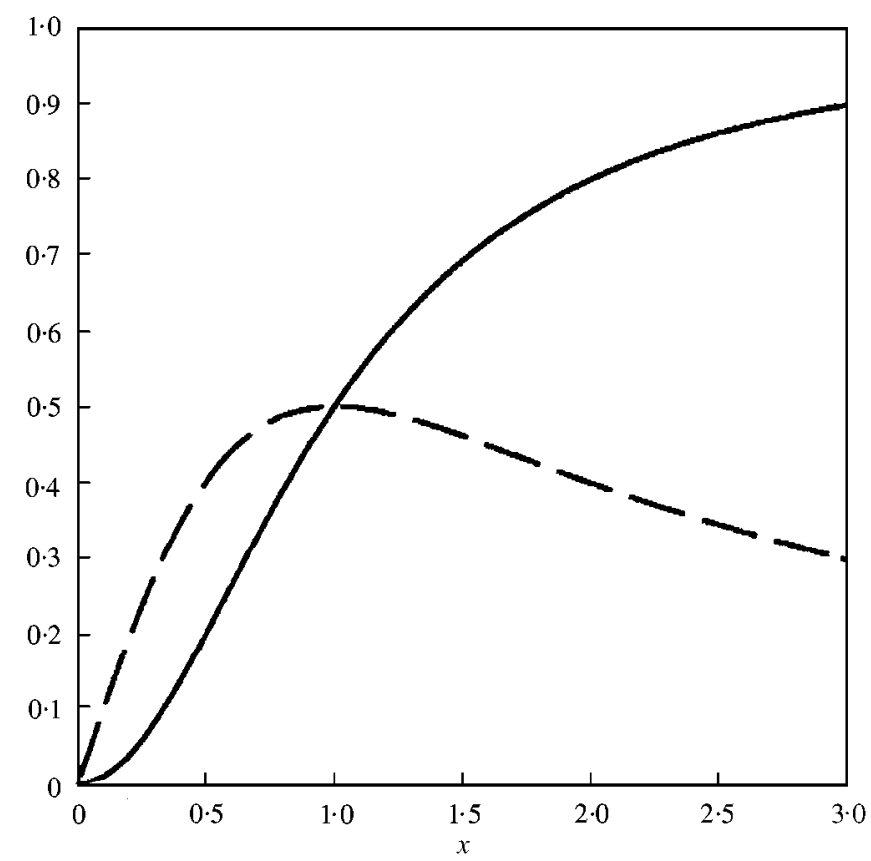

Figure 4. Real (-) and imaginary (- - ) parts of the function representing the change in apparent fluid density due to the plate motion.

vibrational modes of a fluid-saturated sandstone cylinder. In some applications, $\omega_{0}$ can be chosen to coincide with a plate resonance (given by $\Omega$ ) by an appropriate adjustment of the plate dimensions or the clamping conditions.

The porosity, the tortuosity and the permeability which are regarded as macroscopic measurable parameters are closely related to the pore microstructure. The porosity and permeability can have a strong effect on the vibration of a porous plate [6].

\subsection{VISCOUS AND INERTIA FORCES}

The influence of tortuosity has been little studied and is the subject of this section. Figure 5 shows the modulus of the deflection calculated from the present model and plotted as a function of frequency for the water-saturated sandstone. This figure shows that increasing the tortuosity shifts the resonance frequency to lower values and increases the quality factor at the same time, in accordance with the qualitative predictions. As $\tau_{\infty}$ is increased, the resonance frequency and the damping tend to a limit. A physical explanation of this effect in this frequency area is that a higher tortuosity, also called the "drag parameter", corresponds to higher inertia terms so that the apparent dynamic density of the plate is higher. Since according to the classical theory of plates, the resonance frequencies are inversely proportional to the density, a higher density of the porous plate will result in lower resonance frequencies. At the same time, it is reasonable to think that a higher drag parameter is associated with a lower rate of frictional sliding in the pores so that the viscous losses are reduced and the quality factor is enhanced. In other terms, increasing the tortuosity is equivalent to "dragging" or "pushing" more fluid. This can be achieved only if the fluid does not slip along the solid, and if the fluid does not slip along the solid no losses 


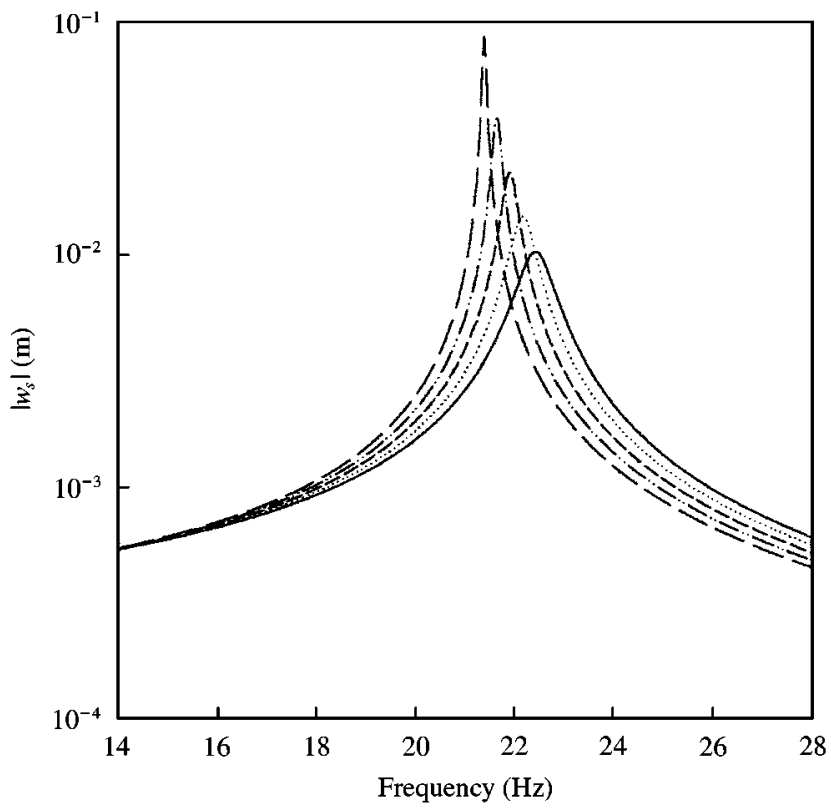

Figure 5. First resonance area of a simply supported porous sandstone for different values of tortuosity: $1 \cdot 0 ; \cdots \cdots, 1 \cdot 2 ;---, 1 \cdot 5 ;-\cdot-, 2 \cdot 0 ;---, 3 \cdot 0$.

by viscous friction occur. The study of the influence of tortuosity shows the relative contributions of the inertia forces and the friction forces. The conclusion that can be drawn here is that an increase of the inertia forces corresponds to a decrease of the friction forces and vice versa. These forces determine the resonance frequencies and the damping.

\subsection{VISCOUS FRICTION IN THE PORES}

In the example of Figure 3, the smooth shape of the curve is largely due to the fairly high structural loss factor of the porous frame (see Table 1). The damping is also partly caused by the viscous friction occurring in the pores during the vibration. The influence of permeability on the damping is studied numerically in this section in the case of the porous sandstone for which a zero loss factor has been taken (see Table 1). It is shown that a frequency for which the damping by viscous friction is maximum exists and is strongly dependent upon the material parameters.

The modulus of the deflection in the vicinity of the lowest resonance frequency has been plotted in Figure 6 as a function of frequency for different values of permeability. For low values of permeability $\left(\kappa=10^{-1} \mathrm{~m}^{2}\right)$, the resonance curve exhibits a sharp peak close to $21 \mathrm{~Hz}$ in this example. The sharpness of the peak implies that the plate and the fluid move practically in phase so that the energy loss by viscous friction in the pores is small. As the permeability is increased, the resonance frequency shifts towards a higher value close to $23 \mathrm{~Hz}$. The peak is also very sharp around this frequency. The interesting point is that an area exists between these two permeabilities where the amplitude of the spectrum decreases dramatically and passes through a minimum. The interpretation of this behaviour is that for each value of permeability, a frequency $\omega_{m}$ exists for which the phase shift between the fluid motion and the solid motion is maximum, the energy loss by viscous friction being 


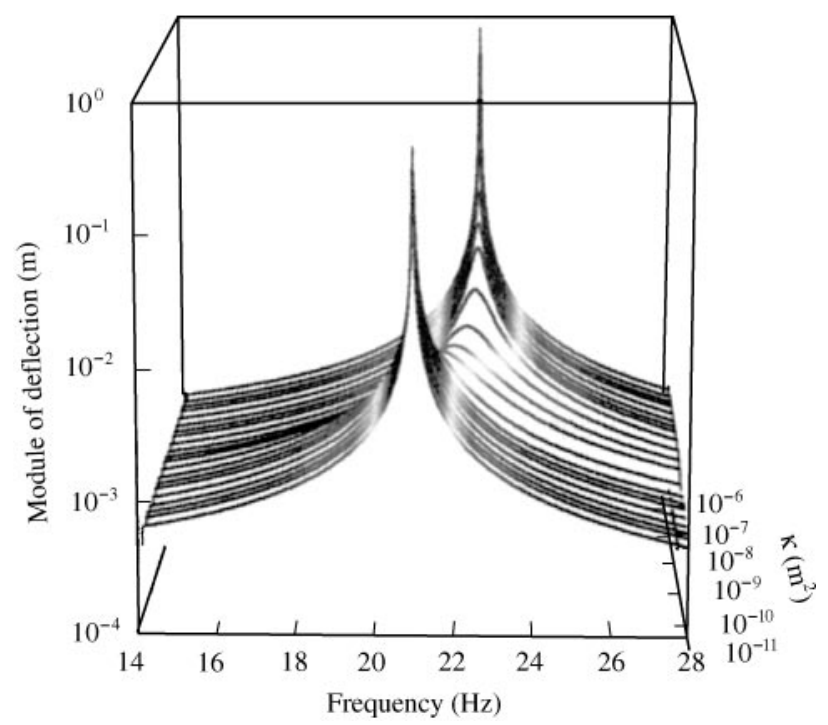

Figure 6. First resonance area of a simply supported porous sandstone for different values of permeability.

maximum at this point. This behaviour occurs only at a single frequency for a given value of permeability. Similar processes can be observed in other resonance areas for other values of permeability. In the example of Figure 6 , a value of $\kappa$ close to $2 \times 10^{-9} \mathrm{~m}^{2}$ will make the frequency of maximum damping coincide with the resonance at around $22 \mathrm{~Hz}$ and renders the peak much smoother. This result can be predicted with a good precision by using the approximate expression for the frequency of maximum damping given by equation (58). A numerical application for three different values of permeability indicates that the damping by viscous friction reaches a maximum at a frequency close to $44 \mathrm{~Hz}$ for $\kappa=10^{-9} \mathrm{~m}^{2} .21 \cdot 7 \mathrm{~Hz}$ for $\kappa=2 \cdot 2 \times 10^{-9} \mathrm{~m}^{2}$ and $16 \mathrm{~Hz}$ for $\kappa=3 \times 10^{-9} \mathrm{~m}^{2}$. Figure 6 shows the maximum damping corresponding to $\kappa=2.2 \times 10^{-9}$. This formula can be used as a tool in some applications to cancel and undesired resonance by making the frequency of maximum damping by viscous friction coincide with a structural resonance of the plate. A physical interpretation of the variation of damping with permeability can be given by a simple argument and by the qualitative conclusions of the preceding section on the relative contributions of the viscous and inertia forces: In the limit of zero permeability, the fluid is totally dragged and no friction occurs so that the energy loss by this mechanism is zero and a sharp peak is predicted. In the limit of infinite permeability, sliding between the solid and the fluid occurs but without energy loss because of the infinite permeability. Between these two limits, Biot's attenuation occurs with a maximum in the function of viscous friction losses.

\section{CONCLUSION}

A theoretical description of the vibration of a porous plate based on the classical theory of homogeneous plates and on Biot's stress-strain relations in porous media has been proposed. For plates thinner than any acoustic wavelength, the assumption of small variations of the amplitude of the relative fluid/solid displacement throughout the thickness can be made and two simple plate equations are found. The first of these characterizes the instantaneous elastic response of the fluid-saturated porous plate while the second describes 
the relative fluid/solid motion, and the delayed after-effect if the complex dissipation term is added. Although it is simpler, the model compares very well with the more rigorous theory of Theodorakopoulos and Beskos [6]. For relatively rigid materials where only the inertial interactions are considered, an approximate calculation of the resonance frequencies has been proposed and the influences of porosity, tortuosity and permeability have been studied. It is found that the resonance frequency is an increasing function of porosity and of permeability, and a decreasing function of tortuosity. The damping factor is an increasing function of porosity and a decreasing function of tortuosity, and it reaches a maximum value at a frequency determined by the three parameters.

\section{ACKNOWLEDGMENTS}

The major part of this work has been carried out at the University of Hull under the British Engineering and Physical Sciences Research Council (EP-SRC) grant number GR/L54905.

The authors are grateful to Professor D. C. Hothersall for his encouragement and to M. J. Swift for sharing his knowledge of re-cycled porous materials.

\section{REFERENCES}

1. M. D. DAhl, E. J. Rice and D. E. GroesbeCK 1990 Journal of the Acoustical Society of America 97, 54-67. Effect of fiber motion on the acoustic behavior of an anisotropic flexible fibrous material.

2. J. F. Allard, C. Depollier, Ph. Guignouard and P. Rebillard 1991 Journal of the Acoustical Society of America 98, 999-1001. Effect of resonance of the frame on the surface impedance of glass wool of high density and stiffness.

3. A. BARdot, B. Brouard and J. F. Allard 1996 Journal of Applied Physics 79, 8223-8229. Frame decoupling at low frequency in thin porous layers saturated by air.

4. M. A BIOT 1956 Journal of the Acoustical Society of America 28, 168-178. Theory of elastic wave propagation in a fluid saturated porous solid. Part I-low frequency range.

5. M. A BIOT 1956 Journal of the Acoustical Society of America 28, 179-191. Theory of elastic wave propagation in a fluid saturated porous solid. Part II-higher frequency range.

6. D. D. Theodorakopoulos and D. E. Beskos 1994 Acta Mechanica 103, 191-203. Flexural vibration of poroelastic plates.

7. M. A. BIOT and D. G. WiLlis 1957 Journal of Applied Mechanics 24, 594-601. The elastic coefficients of the theory of consolidation.

8. M. А ВІот 1962 Journal of Applied Physics 33, 1482-1498. Mechanics of deformation and acoustic propagation in porous media.

9. H. DERESIEWICZ and R. SKALAK 1963 Bulletin of the Seismological Society of America 53, 783-788. On uniqueness in dynamic poroelasticity.

10. S. Timoshenko and S. Woinowsky 1959 Theory of Plates and Shells. New York: McGraw-Hill.

11. W. NowACKI 1963 Dynamics of Elastic Systems. London: Chapman \& Hall.

12. M. A Віот 1964 Journal of Applied Mechanics, American Society of Mechanical Engineers 31, 194-198. Theory of buckling of a porous slab and its thermoelastic analogy.

13. D. L. JOHNSON, J. KOPLIK and R. DASHEN 1987 Journal of Fluid Mechanics 176, 379-402. Theory of dynamic permeability and tortuosity in fluid-saturated porous media.

14. K. Attenborough 1993 Acta Acustica 1, 213-226. Models for the acoustical properties of air-saturated granular media.

15. J. F. Allard 1993 Sound Propagation in Porous Media: Modelling Sound Absorbing Materials. London: Elsevier.

16. K. V. Horoshenkov, K. Attenborough and S. N. Chamdler-Wilde 1998 Journal of the Acoustical Society of America 104, 1198-1209. Padé approximant for the acoustical properties of rigid frame porous media with pore size distribution. 
17. H. Nelisse, O. Beslin and J. Nicolas 1998 Journal of Sound and Vibration 221, 207-225. A generalized approach for the acoustic radiation from a baffled or unbaffled plate with arbitrary boundary conditions, immersed in a light or heavy fluid.

18. I. FAtT 1959 Journal of Applied Mechanics 26, 296-297. The Biot-Willis elastic coefficients for a sandstone.

19. G. B. WARBURTON 1954 Proceedings of the Institution of Mechanical Engineers 168, 371-384. The vibration of rectangular plates.

20. K. J. Dunn 1986 Journal of the Acoustical Society of America 79, 1709-1721. Acoustic attenuation in fluid-saturated porous cylinders at low frequencies. 